\title{
Upgrade Combination Response Is Limited by Prolonged Nucelos(t)ide Analogue Therapy in HBeAg-positive Chronic Hepatitis B: A Real-life Study
}

\author{
Qiaohe Wang, Hu Li, Daohai Ding, Mingli Peng, Hong Ren and Peng Hu* \\ Department of Infectious Diseases, Institute for Viral Hepatitis, The Key Laboratory of Molecular Biology for Infectious Disease, \\ Chinese Ministry of Education, The Second Affiliated Hospital of Chongqing Medical University, Chongqing, China
}

\begin{abstract}
Background and Aims: Few previous studies have reported on a combination response (hepatitis B virus (HBV) DNA undetected, alanine aminotransferase normalization and hepatitis $\mathrm{B}$ e antigen ( $\mathrm{HBeAg}$ ) seroconversion) following nucleos(t) ide analogue (NAs) long-term therapy in patients with chronic hepatitis $B(\mathrm{CHB})$. This study aimed to investigate the combination response on long-term NAs therapy in patients with $\mathrm{HBeAg}$-positive $\mathrm{CHB}$ and to determine whether prolonged therapy is beneficial for combination response, particularly in optimal patients (baseline alanine aminotransferase level $\geq 5$ upper limit of normal and HBV DNA level $<10^{9}$ copies/mL). Methods: In total, $280 \mathrm{HBeAg}$-positive CHB patients were enrolled in this study. Among them, 190 were treated with entecavir and 90 were treated with telbivudine. Results: The cumulative rates of combination response in the total number of patients were $8.6 \%$ at 1 year, $13.2 \%$ at 2 years, $19.1 \%$ at 3 years, $24.2 \%$ at 4 years and $26.0 \%$ at 5 years. In optimal patients, the cumulative rate of combination response was significantly higher than that in the non-optimal patients at 3 years $(p=0.043)$; the trend of the cumulative rate was not strong at the later time. Interestingly, in optimal patients, combination response mainly occurred in the first 3 years. Multivariate analysis identified $\mathrm{HBeAg} /$ anti-HBe seroconversion at 1 year as the only factor for combination response in optimal patients (hazard ratio: $16.321 ; p=0.000)$. During the 3 years, the proportion with aspartate aminotransaminase to platelet ratio index $\leq 0.5$ increased from $15.6 \%$ at baseline to $71.3 \%$ at year 3 . Conclusions: Upgrading the rate of combination response is limited by prolonging the treatment duration of NAs from 3 years to 5 years in $\mathrm{HBeAg}$-positive $\mathrm{CHB}$ patients; a new switch treatment strategy modification should be considered, particularly in optimal patients.
\end{abstract}

Keywords: Chronic hepatitis B; Combination response; Nucleos(t)ide analogue; Long-term therapy.

Abbreviations: ALT, alanine aminotransferase; APRI, aspartate aminotransaminase to platelet ratio index; AST, aspartate aminotransferase; $\mathrm{CHB}$, chronic hepatitis $B$; ETV, entecavir; $\mathrm{HBe}$, hepatitis B e; HBeAg, hepatitis B e antigen; HBV, hepatitis B virus; HCC, hepatocellular carcinoma; LDT, telbivudine; NA, nucleos(t)ide analogue; qPCR, real-time polymerase chain reaction; RCT, randomized controlled trial; ULN, upper limit of normal; VR, virological response.

Received: 10 March 2017; Revised: 4 August 2017; Accepted: 21 August 2017 *Correspondence to: Peng Hu, Department of Infectious Diseases, Institute for Viral Hepatitis, Key Laboratory of Molecular Biology for Infectious Diseases, Chinese Ministry of Education, The Second Affiliated Hospital of Chongqing Medical University, 74 Linjiang Road, Yuzhong District, Chongqing 400010, China. Tel: +86-23-63693289, Fax: +86-23-63703790, E-mail: hp_cq@163.com
Citation of this article: Wang $Q$, Li H, Ding D, Peng M, Ren $\mathrm{H}$, Hu P. Upgrade combination response is limited by prolonged nucelos(t)ide analogue therapy in HBeAg-positive chronic hepatitis B: a real-life study. J Clin Transl Hepatol 2018;6(1):11-17. doi: 10.14218/JCTH.2017.00020.

\section{Introduction}

Worldwide, an estimated 240 million people are chronically infected with hepatitis $B$ virus $(\mathrm{HBV})^{1}$ and have an increased risk of developing cirrhosis and hepatocellular carcinoma (HCC). In China, $22.9 \%$ of patients with liver disease have been found to have chronic $\mathrm{HBV}^{2,3}$

The goal of therapy for CHB is to prevent further development of the disease and progression to cirrhosis, decompensated cirrhosis, end-stage liver disease, HCC and death. The current main drugs for the clinical treatment of $\mathrm{CHB}$ are interferon and nucleos(t)ide analogues (NAs). ${ }^{4}$ In the course of antiviral treatment, studies usually evaluate the efficacy of drugs by virological response and chemical remission. The combination of virological and chemical responses collectively represent a significant marker by which to assess the efficacy of antiviral drugs.

The European Association for the Study of the Liver, Asian Pacific Association for the Study of the Liver, and American Association for the Study of Liver Disease guidelines for treatment recommendations of $\mathrm{CHB}$ advocate that a satisfactory endpoint is a sustained off-therapy virological response with sustained anti-hepatitis $\mathrm{B}$ e ( $\mathrm{HBe}$ ) seroconversion in $\mathrm{HBe}$ antigen (HBeAg)-positive patients. ${ }^{5-7}$ Furthermore, Chinese clinical guidelines recommend a satisfactory endpoint of a sustained off-therapy virological response with sustained anti-HBe seroconversion and alanine aminotransferase (ALT) normality in $\mathrm{HBeAg}$-positive patients. ${ }^{8}$ Therefore, patients may be allowed to stop antiviral therapy if they have achieved a sustained combination of virological and chemical responses.

The previous studies have usually assessed the efficacy of NA treatment for $\mathrm{CHB}$ by achievement of undetectable HBV DNA, HBeAg seroconversion and ALT normality, respectively. Although some investigations have assessed the efficacy of interferon using the combined response, ${ }^{9,10}$ reports of combination responses are not prevalent in the literature, and the characteristics of the combination response remain unknown. Thus, estimates of satisfactory endpoints have not been reliable.

On this basis, the present real-life cohort study aimed to investigate the combination response to long-term NA therapy 
in $\mathrm{HBeAg}$-positive $\mathrm{CHB}$ patients and to identify the characteristics of the combination response.

\section{Methods}

\section{Patients}

The cohort study patients were recruited from $329 \mathrm{HBeAg}-$ positive patients who were followed up between August 2004 and February 2014 at the Department of Infectious Diseases of the Second Affiliated Hospital of Chongqing Medical University (China). This study was a part of the Follow-up and Outcome of Clinical Hepatitis B: A Long-term Study (Focal study). Key inclusion criteria were: (a) ages of $\geq 16$ and $\leq 70$ years; (b) detection of hepatitis B surface antigen, $\mathrm{HBeAg}$ and HBV DNA ( $\geq 10^{5}$ copies $/ \mathrm{mL}$ ) for more than 6 months; (c) ALT $\geq 2$ times the upper limit of the normal (ULN) range or cirrhosis and obvious liver fibrosis detected via non-invasive markers and imaging technique tests in patients with ALT $<2$ ULN. Patients who had infection with hepatitis C virus, hepatitis $D$ virus or human immunodeficiency virus, or had undergone treatment with other antiviral drugs within 6 months were excluded. Moreover, no patients included in the study had used bifendate or bicyclol during their treatment course. The patient treatments were administered according to the Chinese guidelines for preventing and treating chronic hepatitis $B$.

This study was approved by the Ethics Committee of the Second Affiliated Hospital of Chongqing Medical University. Written informed consent was obtained from each patient participant.

\section{Study design}

Eligible patients had been treated with entecavir (ETV) and telbivudine (LDT) monotherapy. Patients could receive adefovir if they had a suboptimal response and exhibited viral breakthrough. All patients underwent clinical examinations, liver function tests and HBV DNA analyses every 3-6 months. All patients included in the analysis participated in at least 48 weeks of follow-up and completed the therapy duration. Patients who did not have data regarding their HBV DNA or $\mathrm{HBeAg}$ levels or who had discontinued treatment because of the combination of other antiviral drugs before 48 weeks were excluded from further analysis.

In the patients who made up the study cohort, the time points of who received another antiviral drug or the switch therapy strategy because of a suboptimal response or viral breakthrough were regarded as the last follow-up endpoint. Finally, according to the baseline HBV DNA and ALT levels, the remaining 280 patients were divided into an optimal patient group and a non-optimal patient group for analysis.

\section{Assay methodology}

Serum hepatitis B surface antigen and anti-hepatitis B surface protein, $\mathrm{HBeAg}$ and anti-HBe, were tested by using aAxSYMmicroparticle enzyme immunoassay (Abbott Laboratories, Abbott Park, IL, USA). HBV DNA levels were measured by a real-time polymerase chain reaction ( $\mathrm{GPCR}$ ) assay using the COBAS TaqMan HBV quantitative test (Roche Molecular Systems, Inc., Branchburg, NJ, USA). Serum aspartate aminotransferase (AST) and ALT were measured by an auto-analyzer
(Roche Analytics; Roche Professional Diagnostics, Penzberg, Germany). The ULN of ALT was defined as $40 \mathrm{IU} / \mathrm{L}$.

Liver cirrhosis was diagnosed by imaging, such as ultrasonography and or computed tomography/magnetic resonance image scanning. The aspartate aminotransaminase to platelet ratio index (APRI) values were calculated using the following formula:

$$
A P R I=\frac{A L T \text { level } /(U L N)}{\text { platelet counts }\left(\frac{10^{9}}{L}\right)} \times 100
$$

An APRI index of $\leq 0.5$ signified the absence of significant fibrosis. APRI index of $\leq 1.0$ signified the absence of cirrhosis. APRI of $>1.5$ signified the presence of significant fibrosis. APRI of $>2$ signified the presence of cirrhosis. APRI scores between 1.0 and 1.5 signified the progressive stages of fibrosis. ${ }^{11,12}$

\section{Evaluation of treatment efficacy and definitions}

The primary endpoint was the cumulative probability of the combination response, which was defined as the collective $\mathrm{HBeAg} /$ anti-HBe seroconversion, HBV DNA <1000 copies/mL, and ALT normalization for the duration of treatment. The secondary endpoints included the cumulative probability of $\mathrm{HBeAg} / \mathrm{anti}-\mathrm{HBe}$ seroconversion, the cumulative probability of the biochemical response, the cumulative probability of the virological response and changing APRI results. Optimal patients were defined as patients with a baseline ALT level of $\geq 5$ ULN and HBV DNA level of $<9 \log _{10}$ copies/MI. ${ }^{13-16}$ Other patients were considered non-optimal patients.

\section{Statistical analysis}

All statistical analyses were performed by the SPSS v.22 software (SPSS, Inc., Chicago, IL, USA). Continuous variables were summarized by the mean, median, minimum and maximum values. Binary variables were summarized by counts and percentages. Baseline characteristics were compared by $\chi^{2}$ tests (for dichotomous variables) and the 2-sample $t$-test with unequal variances (for quantitative variables). KaplanMeier estimators were used to present the cumulative incidence of the combination response, $\mathrm{HBeAg} / \mathrm{Anti}-\mathrm{HBe}$ seroconversion, ALT normality and undetectable HBV-DNA, and differences were determined using the log-rank test. A two-sided $P$-value of $<0.05$ was considered significant for all statistical tests.

\section{Results}

\section{Patient characteristics}

We identified $329 \mathrm{HBeAg}$-positive patients treated with ETV and LDT monotherapy. Thirty-nine patients were excluded due to missing data regarding HBV DNA and HBeAg for the follow-up duration, and 10 patients were excluded because of switch to another treatment protocol before 48 weeks. Hence, in total, 280 patients were included, consisting of 51 patients who were optimal and 229 who were non-optimal.

We followed 280, 252, 176, 127 and 87 patients for 48, 96 144, 192 and 240 weeks, respectively (Fig. 1). Only one non-optimal patient progressed to HCC. The baseline characteristics of the patients were compared by optimal/non-optimal 
Wang Q. et al: Prolonged NA therapy in $\mathrm{HBeAg}+\mathrm{CHB}$

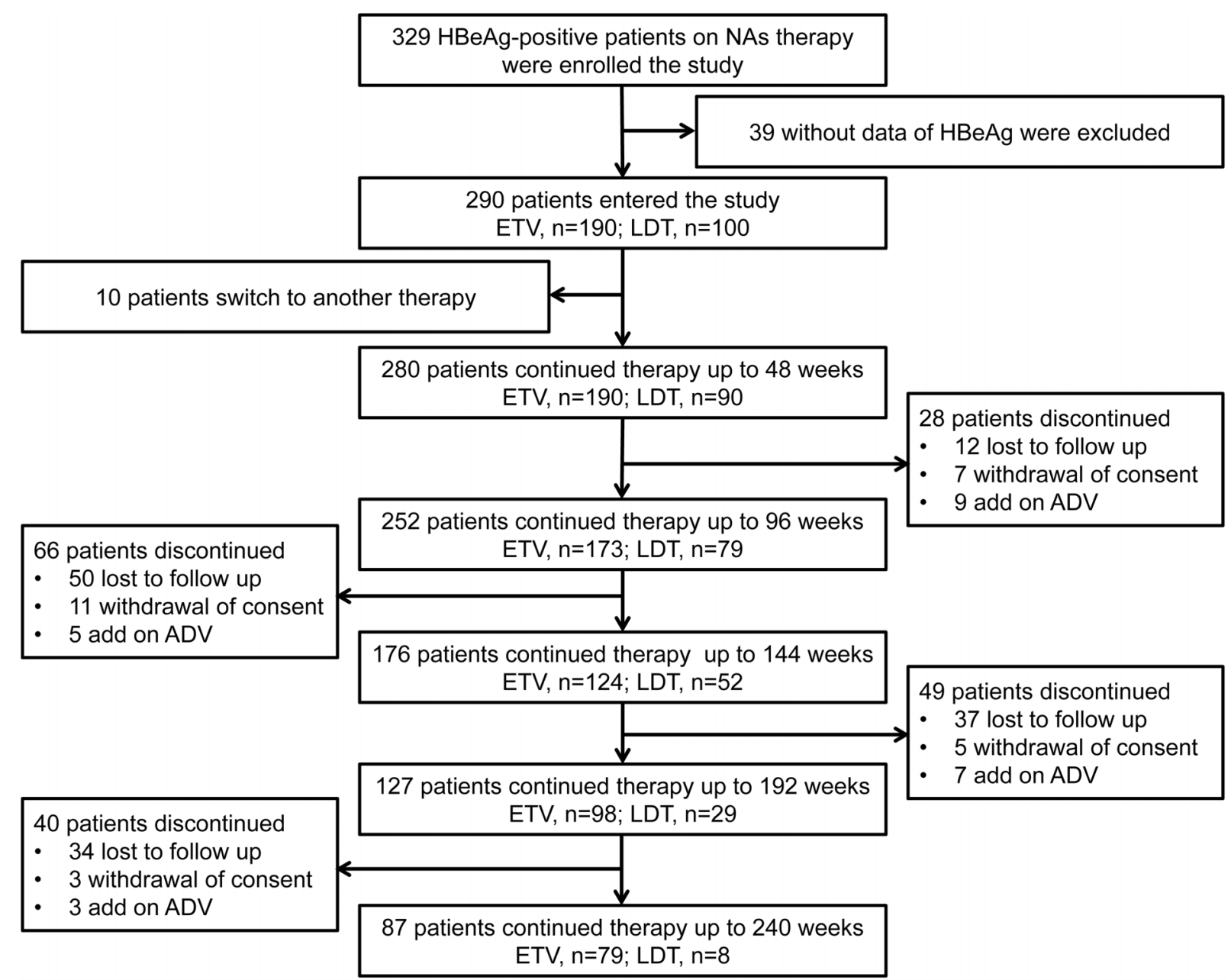

Fig. 1. Flowchart showing the enrollment and exits of patients during the course of treatment. Abbreviations: ADV, adefovir; ETV, entecavir; HBeAg, hepatitis B e antigen; LDT, telbivudine; NAs, nucleos(t)ide analogues.

grouping (Table 1$)$. The patients in the optimal group had a higher level of serum HBV DNA $(p=0.012)$ and ALT $(p=$ 0.000 ) than did the non-optimal group, whereas there were no significant differences in their sex, age or presence of cirrhosis.

\section{Combination response rates were low among the total patients following long-term NA therapy}

A Kaplan-Meier plot was used to analyze the cumulative rates of combination response, and the differences were calculated by the log-rank test. The cumulative rates of combination response in the total patients were $8.6 \%$ at 48 weeks, $13.2 \%$ at 96 weeks, $19.1 \%$ at 144 weeks, $24.2 \%$ at 192 weeks and $26.0 \%$ at 240 weeks. The rates were lower than the cumulative rates of $\mathrm{HBeAg} / \mathrm{anti}-\mathrm{HBe}$ seroconversion, $\mathrm{ALT}$ normality and undetectable HBV DNA (Fig. 2).

The combination response rates of patients between LDT and ETV treatment were evaluated. The combination response rates of the LDT-treated patients were higher than those of the ETV-treated patients. The cumulative rates of combination response were $15.6 \%$ versus $5.3 \%$ at 48 weeks, $30.2 \%$ versus $13.8 \%$ at 144 weeks, and $32.6 \%$ versus $22.1 \%$ at 240 weeks, respectively.

\section{Combination response occurred mainly during the first 3 years}

In optimal patients, the 144-week cumulative rate of combination response was significantly higher than that in the nonoptimal patients $(p=0.042)$, and the trend of the cumulative rate was not strong at the later time (Fig. 3). The cumulative rates of combination response in the optimal and non-optimal groups were $27.4 \%$ versus $17.6 \%$ at 144 weeks and $27.4 \%$ versus $25.4 \%$ at 240 weeks, respectively. Interestingly, in optimal patients, the combination response mainly occurred during the first 3 years (Fig. 4). The cumulative rates of combination response increased $8.6 \%$ from year 1 to year 2 and $3.1 \%$ from year 2 to year 3 . The growth trend of the combination response clearly descended, with no incremental after 3 years.

\section{Impact factors associated with the combination response in optimal patients}

In optimal patients, the combination response mainly occurred during the first 3 years; that is, prolonged therapy duration of NAs from 3 years to 5 years could not achieve a combination response for optimal patients with $\mathrm{HBeAg}$-positive $\mathrm{CHB}$ 


\begin{tabular}{lllll}
\hline & & \multicolumn{2}{l}{ Entire cohort } & \\
\cline { 4 - 5 } Variable & Total patients, $n=280$ & Optimal group, $n=51$ & Non-optimal group, $n=229$ & $P$ \\
\hline Male, $n(\%)$ & $216(77.1)$ & $39(76.5)$ & $177(68.5)$ & $56(24.5)$ \\
Cirrhosis, $n(\%)$ & $69(24.6)$ & $13(25.5)$ & $30.36 \pm 7.99$ \\
Age in years & $31.88 \pm 8.57$ & $31.88 \pm 8.57$ & $8.17 \pm 1.34$ & 0.899 \\
HBV-DNA levels, as $\log _{10}$ copies/mL & $8.81 \pm 1.30$ & $7.67 \pm 0.98$ & $95(2-586)$ & 0.226 \\
ALT, as U/L & $108(2-841)$ & $258(201-841)$ & & 0.012 \\
Drug, $n(\%)$ & & & $72(31.4)$ \\
$\quad$ Telbivudine & $90(32.1)$ & $18(35.3)$ & $157(68.6)$ \\
$\quad$ Entecavir & $190(67.9)$ & $33(64.7)$ &
\end{tabular}

patients. The multivariate analysis demonstrated that $\mathrm{HBeAg} /$ anti-HBe seroconversion at 1 year was the only determining factor for the combination response (hazard ratio: 16.321; $p=0.000$ ) (Table 2). Similarly, the same trend was evident for $\mathrm{HBeAg} / \mathrm{anti}-\mathrm{HBe}$ seroconversion.
Hepatic fibrosis obviously improved after long-term NA treatment

In total, 160 patents had available complete dates of APRI from baseline to year 3 of follow-up. After 3 years of NA

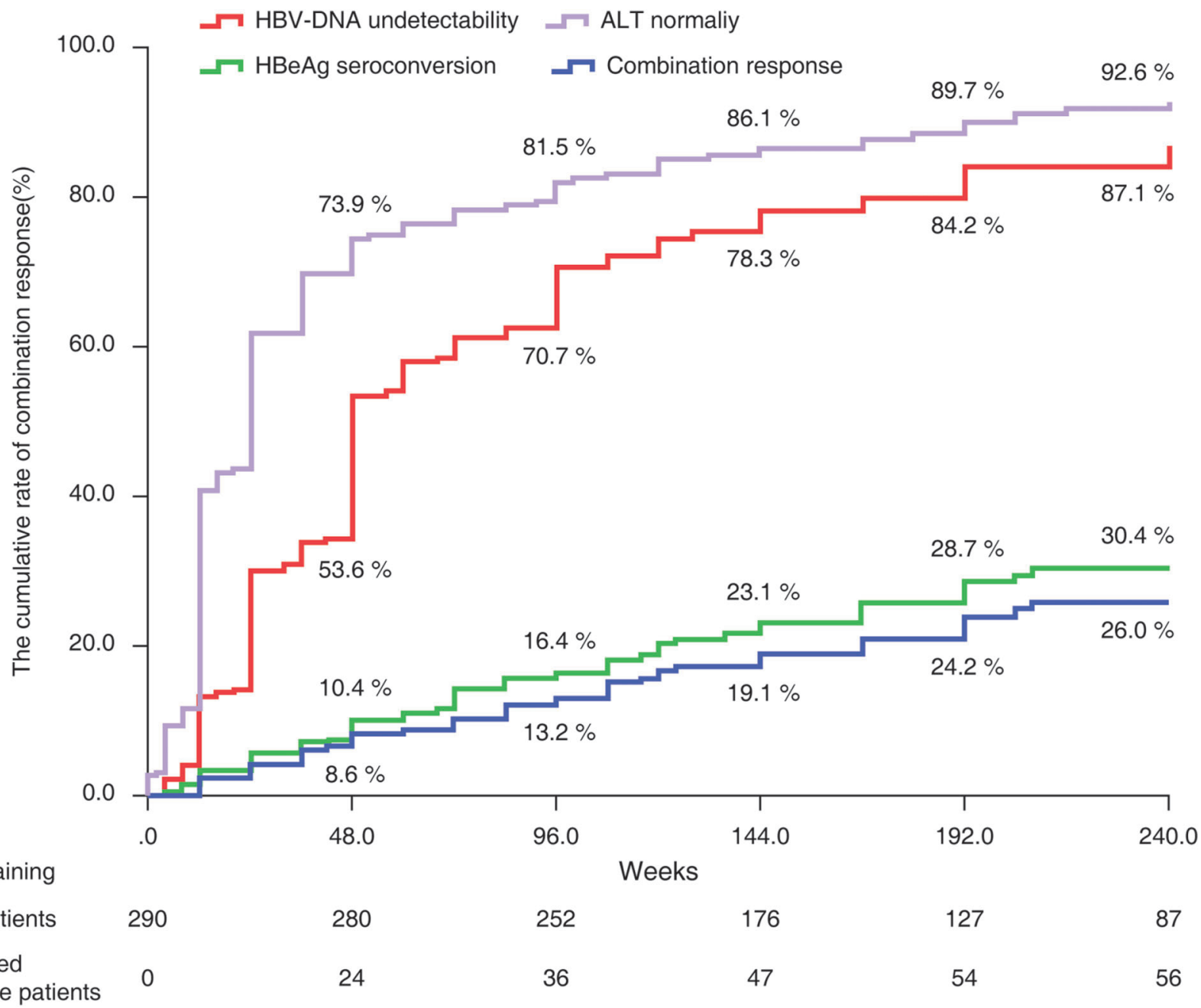

No remaining

Total patients

Combined response patients

Fig. 2. Effects of LDT and ETV on the combination response. The cumulative combination response rates were estimated by the Kaplan-Meier method. Abbreviations: ETV, entecavir; LDT, telbivudine. 
Wang Q. et al: Prolonged NA therapy in $\mathrm{HBeAg}+\mathrm{CHB}$

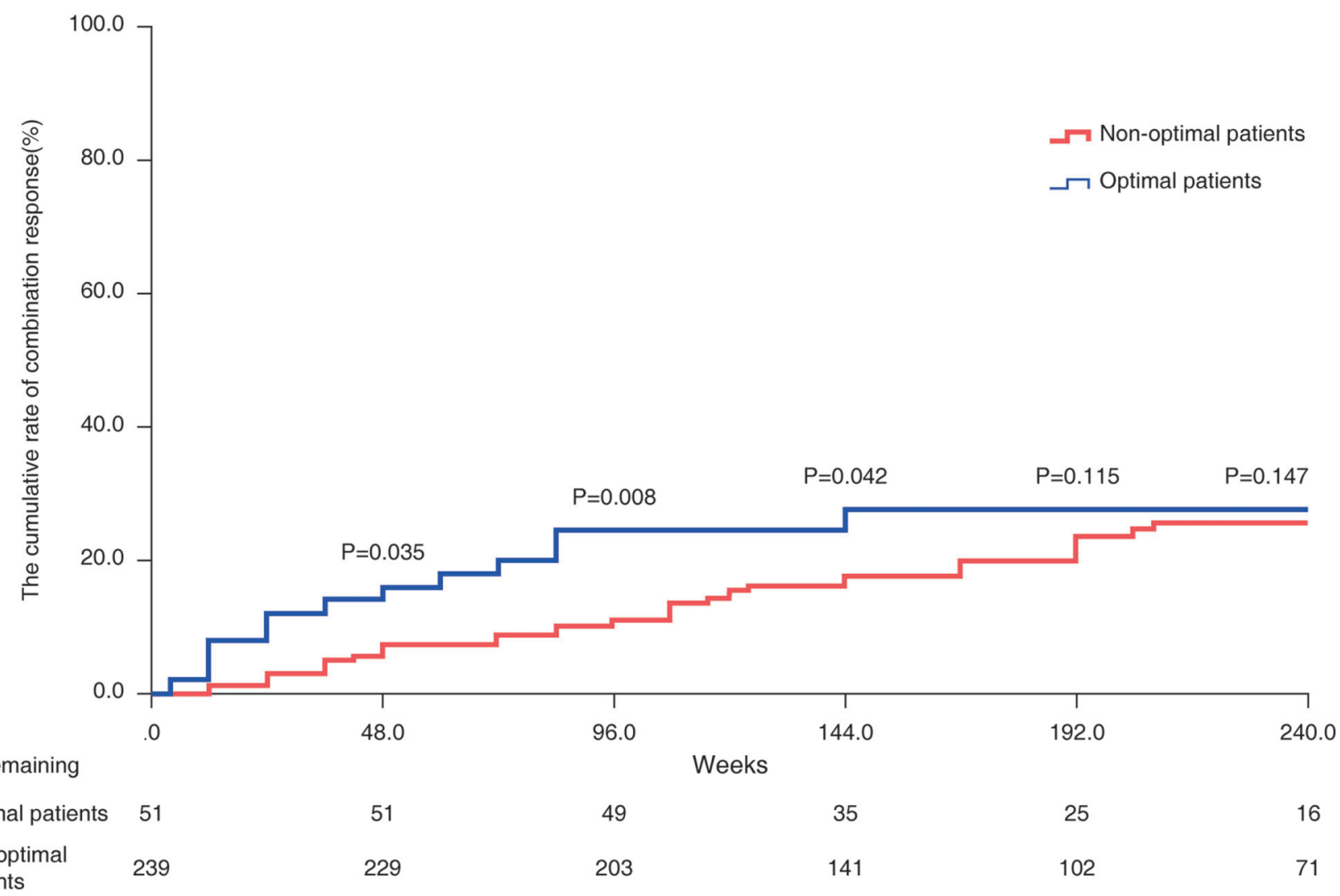

Fig. 3. Combination response in patient subgroups, stratified by baseline ALT and HBV DNA levels. Cumulative combination response rates were estimated by the Kaplan-Meier method, and the differences were supported by the log-rank test. Abbreviation: ALT, alanine transaminase.

treatment duration, the proportions of patients with APRI were changed (Fig. 5). The proportions with APRI of $\leq 0.5$ increased from $15.6 \%(25 / 160)$ at baseline to $71.3 \%(114 / 160)$ at year 3 , and APRI of $>1.5$ decreased from $43.8 \%(70 / 160)$ at baseline to $1.9 \%(3 / 160)$ at year 3 .

\section{Discussion}

Our study has shown that with up to 5 years of ETV and LDT monotherapy, the cumulative rate of combination response

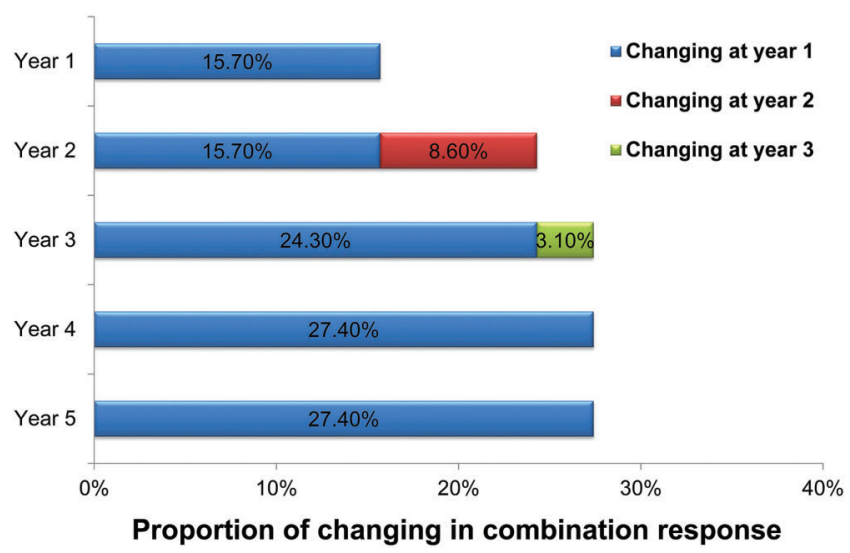

Fig. 4. Combination response occurred mainly during the first 3 years in optimal patients. was only $26.0 \%$. The rate was lower than the cumulative rates of $\mathrm{HBeAg} / \mathrm{anti}-\mathrm{HBe}$ seroconversion, undetectable $\mathrm{HBV}$ DNA and ALT normality, respectively, in previous clinical trials. One previous clinical study indicated that the 240-week cumulative rates of $\mathrm{HBeAg} / \mathrm{anti}-\mathrm{HBe}$ seroconversion upon treatment with ETV and LDT were $36 \%$ and 53\%, respectively. ${ }^{13}$ Another study showed that the $\mathrm{HBeAg} /$ anti-HBe seroconversion rate was $36.7 \%$ with 5 -year ETV treatment in $\mathrm{HBeAg}$-positive CHB. ${ }^{17}$ In an ETV 7-year treatment study, $\mathrm{HBeAg} /$ anti-HBe seroconversion rates at years 3 and 7 were $26.7 \%$ and $37.5 \%$, respectively. ${ }^{18}$ Additionally, many investigations have shown that ETV and LDT have a higher rate of $\mathrm{HBeAg} / \mathrm{anti}-\mathrm{HBe}$ seroconversion in patients with HBeAg-positive CHB. ${ }^{19-24}$ Therefore, in an analysis of the efficacy of long-term antiviral drug therapy, the combination response showed no strong trend with the virological response or chemical remission.

Similarly, in optimal patients, combination response rates were low, and the combination response and $\mathrm{HBeAg} / \mathrm{anti}-\mathrm{HBe}$ seroconversion mainly occurred during the first 3 years; prolonged therapy after 3 years is limited for the combination response and $\mathrm{HBeAg} / \mathrm{anti}-\mathrm{HBe}$ seroconversion. The same result was reported in a head-to head study. ${ }^{13}$ In other words, new switch-treatment strategies are necessary to pursue a greater combination response after 3 years of NA treatment in optimal patients with baseline HBV DNA levels $<9 \log _{10}$ copies/mL and ALT $\geq 5$ ULN. This finding is clinically significant and may be used to guide treatment decisions and avoid unnecessary treatment of optimal patients. In addition, 
Wang Q. et al: Prolonged NA therapy in $\mathrm{HBeAg}+\mathrm{CHB}$

Table 2. Univariate and multivariate analyses for factors associated with combination response in optimal patients on NA therapy

\begin{tabular}{|c|c|c|c|c|c|}
\hline \multirow[b]{2}{*}{ Variable } & \multicolumn{2}{|l|}{ Value of patients } & \multirow{2}{*}{$\begin{array}{l}\text { Univariate } \\
P\end{array}$} & \multicolumn{2}{|l|}{ Multivariate } \\
\hline & $\begin{array}{l}\text { With combination } \\
\text { response, } n=13\end{array}$ & $\begin{array}{l}\text { Without combination } \\
\text { response, } n=38\end{array}$ & & $\mathrm{HR}(95 \% \mathrm{CI})$ & $P$ \\
\hline Male, $n(\%)$ & $8(61.5)$ & $31(81.6)$ & 0.127 & & \\
\hline Age in years & $33.54 \pm 10.74$ & $31.32 \pm 7.77$ & 0.301 & & 0.720 \\
\hline $\begin{array}{l}\text { HBV DNA, as } \log _{10} \\
\text { copies/mL }\end{array}$ & $7.57 \pm 1.19$ & $7.70 \pm 0.92$ & 0.628 & & 0.620 \\
\hline $\mathrm{ALT}$, as U/L & $270(207-841)$ & $250(201-477)$ & 0.103 & & 0.148 \\
\hline $\mathrm{VR}^{*}$ at $\mathrm{yr} 1, n(\%)$ & $7(53.8)$ & $21(55.3)$ & 0.938 & & 0.723 \\
\hline $\begin{array}{l}\text { HBeAg seroconversion } \\
\text { at yr } 1, n(\%)\end{array}$ & $8(61.5)$ & $2(5.3)$ & 0.000 & $16.321(4.472-59.563)$ & 0.000 \\
\hline
\end{tabular}

* undetectable HBV DNA (HBV DNA $<1.0 \times 10^{3}$ copies $/ \mathrm{mL}$ ) by sensitive PCR assay at year 1 .

Abbreviations: ALT, alanine aminotransaminase; $\mathrm{HBeAg}$, hepatitis $\mathrm{B}$ e antigen; $\mathrm{HR}$, hazard ratio.

further studies with a larger sample size are needed to confirm the conclusion.

In optimal patients, the cumulative rate of $\mathrm{HBeAg} / \mathrm{anti}-$ $\mathrm{HBe}$ seroconversion was significantly higher than that of nonoptimal patients, which indicates that baseline HBV DNA $<9 \log _{10}$ copies/mL and ALT $\geq 5$ ULN may contribute to the higher rate of $\mathrm{HBeAg}$ seroconversion. This finding confirms previously reported relationships between $\mathrm{HBeAg}$ seroconversion and baseline HBV DNA and ALT levels. It also agrees with previous reports of baseline ALT $>5$ ULN as a positive predictor for $\mathrm{HBeAg}$ seroconversion, ${ }^{13,14}$ and serum HBV DNA $<9 \log _{10}$ copies/mL as a strong predictor for better virological outcomes. ${ }^{13-17}$

The present study showed that $\mathrm{HBeAg} / \mathrm{anti}-\mathrm{HBe}$ seroconversion at 1 year was the only determining factor of combination response in optimal patients, which demonstrated that the sooner HBeAg/anti-HBe seroconversion occurs, the easier the combination response will be in optimal patients who have $\mathrm{HBeAg}$-positive CHB during NA treatment.

After 3 years of LDT and ETV treatment, the proportion with APRI $\leq 0.5$ increased from $15.6 \%$ at baseline to $71.3 \%$ at year 3, and APRI $>1.5$ decreased from $43.8 \%$ at baseline to $1.9 \%$ at year 3 . APRI, as a marker of hepatic fibrosis, was significantly improved. In other words, the hepatic fibrosis

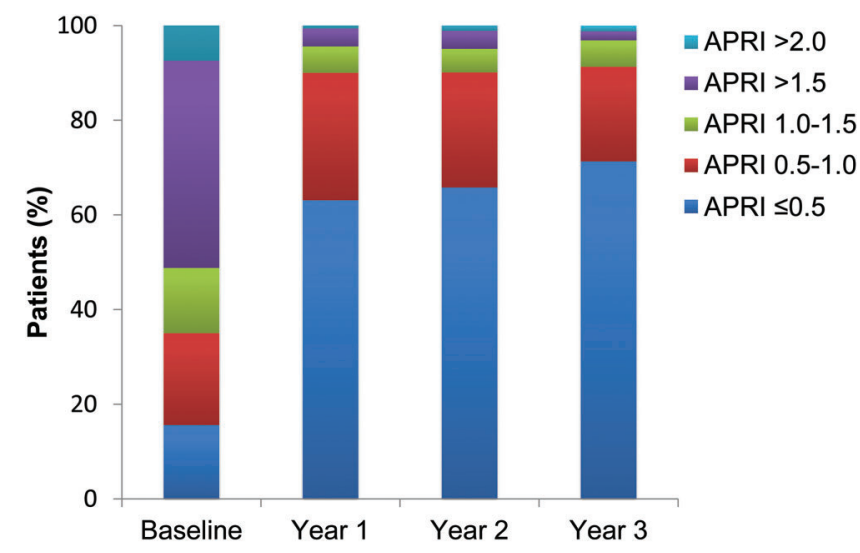

Fig. 5. Changing results of APRI over the 3-year therapy course. Abbreviation: APRI, aspartate aminotransaminase to platelet ratio index. had obviously improved after the 3-year NA treatment, and this finding was similar to those of previous studies. ${ }^{23,25-27}$

The present study had limitations. First, it was not a randomized controlled trial in design, and it was conducted only with Chinese patients. Second, the optimal sample size was small and comprised of young individuals. Therefore, the conclusions should be confirmed by further studies from multicenter and randomized cohorts.

In conclusion, prolonged therapy after 3 years is limited for the combination response to 5-year NA treatment in optimal patients with HBeAg-positive CHB. Consequently, new switch-treatment strategies are required.

\section{Acknowledgments}

The authors thank the patients, investigators and study centers for their participation. This study was supported in part by grants from the National Natural Science Foundation of China (Nos. 30930082, 81171561 and 30972584) and the National Science and Technology Major Project of China (Nos. 2008ZX10002-006, 2012ZX10002007001, 2017ZX10202203007 and 2017ZX10202203-008).

\section{Conflict of interest}

The authors have no conflict of interests related to this publication.

\section{Author contributions}

Contributed to the study design, data collection and analysis, as well as performing the research and drafting the manuscript (QW), statistical analysis (HL), data collection (DD), study design and revision of the manuscript (MP, HR), responsible for the entire study design, data analysis and manuscript revision $(\mathrm{PH})$. All authors have approved the final version of the manuscript.

\section{References}

[1] Ott J], Stevens GA, Groeger ], Wiersma ST. Global epidemiology of hepatitis $B$ virus infection: new estimates of age-specific HBsAg seroprevalence and endemicity. Vaccine 2012;30:2212-2219. doi: 10.1016/j.vaccine.2011.12. 116. 
[2] Wang FS, Fan JG, Zhang Z, Gao B, Wang HY. The global burden of liver disease: the major impact of China. Hepatology 2014;60:2099-2108. doi: 10.1002/hep. 27406 .

[3] Tujios SR, Lee WM. New advances in chronic hepatitis B. Curr Opin Gastroenterol 2012;28:193-197. doi: 10.1097/MOG.0b013e32835297ef.

[4] Chen $\mathrm{CH}$, Lin $\mathrm{CL}$, Hu TH, Hung CH, Tseng PL, Wang JH, et al. Entecavir vs. lamivudine in chronic hepatitis $\mathrm{B}$ patients with severe acute exacerbation and hepatic decompensation. J Hepatol 2014;60:1127-1134. doi: 10.1016/j. jhep.2014.02.013.

[5] European Association For The Study Of The Liver. EASL clinical practice guidelines: Management of chronic hepatitis B virus infection. J Hepatol 2012;57:167-185. doi: 10.1016/j.jhep.2012.02.010.

[6] Sarin SK, Kumar M, Lau GK, Abbas Z, Chan HL, Chen CJ, et al. Asian-Pacific clinical practice guidelines on the management of hepatitis B: a 2015 update. Hepatol Int 2016;10:1-98. doi: 10.1007/s12072-015-9675-4.

[7] Merriman RB, Tran TT. AASLD practice guidelines: The past, the present, and the future. Hepatology 2016;63:31-34. doi: 10.1002/hep.28345.

[8] Chinese Society of Hepatology, Chinese Medical Association; Chinese Society of Infectious Diseases, Chinese Medical Association, Hou JL, lai W. The guideline of prevention and treatment for chronic hepatitis B: a 2015 update. Zhonghua Gan Zang Bing Za Zhi 2015;23:888-905. doi: 10.3760/cma.j. issn.1007-3418.2015.12.002.

[9] Xie Q, Zhou H, Bai X, Wu S, Chen J], Sheng J, et al. A randomized, open-label clinical study of combined pegylated interferon Alfa-2a (40KD) and entecavir treatment for hepatitis B " $\mathrm{e}$ " antigen-positive chronic hepatitis B. Clin Infect Dis 2014;59:1714-1723. doi: 10.1093/cid/ciu702.

[10] Lau GK, Piratvisuth T, Luo KX, Marcellin P, Thongsawat S, Cooksley G, et al. Peginterferon Alfa-2a, lamivudine, and the combination for $\mathrm{HBeAg}$-positive chronic hepatitis B. N Engl J Med 2005;352:2682-2695. doi: 10.1056/ NEJMoa043470.

[11] Wai CT, Greenson JK, Fontana RJ, Kalbfleisch JD, Marrero JA, Conjeevaram $\mathrm{HS}$, et al. A simple noninvasive index can predict both significant fibrosis and cirrhosis in patients with chronic hepatitis C. Hepatology 2003;38:518-526. doi: 10.1053/jhep.2003.50346.

[12] Cross TJ, Calvaruso V, Foxton MR, Manousou P, Quaglia A, Grillo $F$, et al. A simple, noninvasive test for the diagnosis of liver fibrosis in patients with hepatitis C recurrence after liver transplantation. J Viral Hepat 2010;17: 640-649. doi: 10.1111/j.1365-2893.2009.01222.x.

[13] Zhang $Y$, Hu P, Qi X, Ren H, Mao RC, Zhang JM. A comparison of telbivudine and entecavir in the treatment of hepatitis $B$ e antigen-positive patients: a prospective cohort study in China. Clin Microbiol Infect 2016;22:287. e1-e9. doi: 10.1016/j.cmi.2015.10.024.

[14] Zeuzem S, Gane E, Liaw YF, Lim SG, DiBisceglie A, Buti M, et al. Baseline characteristics and early on-treatment response predict the outcomes of 2 years of telbivudine treatment of chronic hepatitis B. J Hepatol 2009;51: 11-20. doi: 10.1016/j.jhep.2008.12.019.

[15] Perrillo RP, Lai CL, Liaw YF, Dienstag JL, Schiff ER, Schalm SW, et al. Predictors of HBeAg loss after lamivudine treatment for chronic hepatitis B. Hepatology 2002;36:186-194. doi: 10.1053/jhep.2002.34294.
[16] Janssen $\mathrm{HL}$, van Zonneveld $\mathrm{M}$, Senturk $\mathrm{H}$, Zeuzem $\mathrm{S}$, Akarca US, Cakaloglu $\mathrm{Y}$, et al. Pegylated interferon alfa-2b alone or in combination with lamivudine for HBeAg-positive chronic hepatitis B: a randomised trial. Lancet 2005;365: 123-129. doi: 10.1016/S0140-6736(05)17701-0.

[17] Liu F, Zou F, Wang X, Hu H, Hu P, Ren H. A model with combined viral and metabolic factors effectively predicts HBeAg status under long term entecavir therapy: a prospective cohort study. Virol J 2015;12:179. doi: 10. 1186/s12985-015-0409-y.

[18] Lee HW, Kwon JC, Oh IS, Chang HY, Cha YJ, Choi IS, et al. Prolonged entecavir therapy is not effective for $\mathrm{HBeAg}$ seroconversion in treatment-naive chronic hepatitis $B$ patients with a partial virological response. Antimicrob Agents Chemother 2015;59:5348-5356. doi: 10.1128/AAC. 01017-15.

[19] Ridruejo E, Adrover R, Cocozzella D, Reggiardo MV, Estepo C, Schroder T, et al. Effectiveness of entecavir in chronic hepatitis B NUC-naive patients in routine clinical practice. Int J Clin Pract 2011;65:866-870. doi: 10.1111/j. 1742-1241.2011.02719.x.

[20] Luo J, Li X, Wu Y, Lin G, Pang Y, Zhang X, et al. Efficacy of entecavir treatment for up to 5 years in nucleos(t)ide-naïve chronic hepatitis $B$ patients in real life. Int J Med Sci 2013;10:427-433. doi: 10.7150/ijms.5472.

[21] Wang CC, Tseng KC, Peng CY, Hsieh TY, Lin CL, Su TH, et al. Viral load and alanine aminotransferase correlate with serologic response in chronic hepatitis B patients treated with entecavir. J Gastroenterol Hepatol 2013;28: 46-50. doi: 10.1111/j.1440-1746.2012.07269.x.

[22] Liaw YF, Gane E, Leung N, Zeuzem S, Wang Y, Lai CL, et al. 2-Year GLOBE trial results: telbivudine Is superior to lamivudine in patients with chronic hepatitis B. Gastroenterology 2009;136:486-495. doi: 10.1053/j.gastro.2008. 10.026 .

[23] Hou JL, Xu D, Shi G, Wan M, Goodman Z, Tan D, et al. Long-term telbivudine treatment results in resolution of liver inflammation and fibrosis in patients with chronic hepatitis B. Adv Ther 2015;32:727-741. doi: 10.1007/s12325015-0232-2.

[24] Wang Y, Thongsawat S, Gane EJ, Liaw YF, Jia J, Hou J, et al. Efficacy and safety of continuous 4-year telbivudine treatment in patients with chronic hepatitis B. J Viral Hepat 2013;20:e37-e46. doi: 10.1111/jvh.12025.

[25] Schiff ER, Lee SS, Chao YC, Kew Yoon S, Bessone F, Wu SS, et al. Long-term treatment with entecavir induces reversal of advanced fibrosis or cirrhosis in patients with chronic hepatitis B. Clin Gastroenterol Hepatol 2011;9:274276. doi: $10.1016 /$ j.cgh.2010.11.040.

[26] Chang TT, Liaw YF, Wu SS, Schiff E, Han KH, Lai CL, et al. Long-term entecavir therapy results in the reversal of fibrosis/cirrhosis and continued histological improvement in patients with chronic hepatitis B. Hepatology 2010;52:886893. doi: 10.1002/hep.23785.

[27] Marcellin P, Gane E, Buti M, Afdhal N, Sievert W, Jacobson IM, et al. Regression of cirrhosis during treatment with tenofovir disoproxil fumarate for chronic hepatitis B: a 5-year open-label follow-up study. Lancet 2013;381: 468-475. doi: 10.1016/S0140-6736(12)61425-1. 